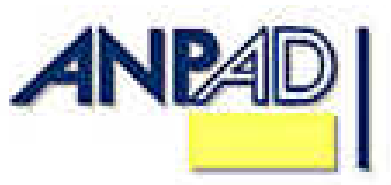

Disponível em http://www.anpad.org.br/rac

RAC, Curitiba, v. 13, n. 2, art. 8, p. 310-327, Abr./Jun. 2009

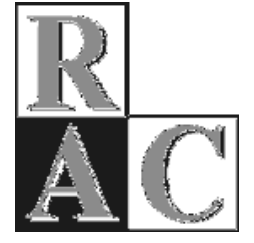

\title{
Proposta de Escala para Mensurar o Valor Percebido no Varejo Bancário Brasileiro
}

\section{The Customer Value Structure in the Brazilian Personal Retail Banking Market: a Specific Summated Rating Scale}

João Carlos Neves de Paiva *

Doutorando em Administração pela UnB. Executivo do Banco do Brasil S.A., Cidade do Panamá, Panamá.

Francisco Vidal Barbosa Ph.D. pela Aston University, Birmingham, Reino Unido. Professor da FACE/UFMG, Belo Horizonte/MG, Brasil.

Áurea Helena Puga Ribeiro Doutora em Administração pela FGV/SP. Professora e pesquisadora da FDC, Nova Lima/MG, Brasil.

* Endereço: João Carlos Neves de Paiva

Av. Aquilino de La Guardia y Calle 47, Ed. Ocean Business Plaza, oficina 1202, Cidade do Panamá, Panamá, 0823-01180. E-mail: joaocarlos@ bb.com.br

Copyright (C 2009 RAC. Todos os direitos, inclusive de tradução, são reservados. É permitido citar parte de artigos sem autorização prévia desde que seja identificada a fonte. 


\section{RESUMO}

O objetivo deste estudo foi desenvolver uma escala para identificar e classificar os atributos mais valorizados pelos clientes no relacionamento com bancos de varejo. Há carência de estudos sobre a percepção de valor que considerem variáveis como setores da economia, segmentos de consumidores ou regiões geográficas, possibilitando maior precisão nas estratégias para conquistar, de forma duradoura, a preferência de consumo do cliente, focando seus negócios atuais e futuros. A metodologia utilizada reuniu procedimentos sugeridos por diversos autores que são referências na área, com ênfase no processo de triangulação, em que a abordagem qualitativa funciona como suporte consistente para análises quantitativas. A escala passou por processos de purificação, diversos testes qualitativos e quantitativos, apresentando confiabilidade e validade. Os resultados indicaram que escalas específicas são mais eficazes para explicar fenômenos de marketing relacionados a valor para o cliente, bem como a relevância da metodologia utilizada para novas pesquisas nesse campo de estudos. Contudo são recomendáveis novos estudos empíricos para confirmar a validade da escala proposta.

Palavras-chave: marketing de serviços; valor para o cliente; comportamento do consumidor; escala; bancos de varejo.

\section{ABSTRACT}

The aim of this study is to develop a scale in order to identify and classify the most valued attributes of retail banks by their customers. There is a shortage of studies about customer value that take into account variables such as economic sectors, customer segments or geographic regions, thereby allowing greater precision on developing strategies to conquer customer loyalty in present and future transactions. The methodology applied relies on solid conceptual and theoretical thinking and gathered procedures recommended by recognized authors, including the triangulation process, which emphasizes the qualitative approach as a support for quantitative analysis. Purification processes and several qualitative and quantitative tests provide reliability and validity to the scale. The results also show that specific scales are more precise when it comes to explaining marketing phenomena related to customer value in addition to the relevance of the methodology for further studies in that field. This notwithstanding, further studies are recommended in order to confirm the validity of the proposed scale.

Key words: service marketing; customer value; customer behavior; scale, retail banking. 


\section{INTRODUÇÃO}

$\mathrm{Na}$ evolução das relações mercadológicas constatou-se que, além de atrair, é necessário conquistar a lealdade do cliente, fator determinante para o sucesso dos fornecedores de bens e serviços. Reichheld (1996) detectou que empresas que conseguiam atingir patamares elevados de lealdade e retenção de clientes obtinham lucros mais expressivos e consistentes, além de crescimento mais rápido. A partir dessa constatação, desenvolveu estratégias organizacionais para aumentar a lealdade dos clientes, cuja operacionalização se baseia no provimento ao cliente de benefícios que ele percebe como significativos e vantajosos, considerando, até mesmo as ofertas dos concorrentes. Ao entender como relevantes esses diferenciais, o cliente se torna leal à empresa, retribuindo com a repetição de compras em longo prazo. Acrescenta esse autor que, quando uma companhia entrega, consistentemente, um valor superior ao cliente, conquista a sua lealdade e vê aumentada a sua participação de mercado e a lucratividade, enquanto cai o custo de aquisição de novos consumidores e da prestação de serviços. Nesse sentido, cresce a importância de se saber o que as pessoas percebem como valioso, a fim de que uma empresa possa ir além da aquisição e da retenção do cliente, tendo em vista o incremento das relações negociais e mensuração do seu retorno financeiro atual e futuro. Esse quadro pode ser observado no mercado bancário de varejo, onde há intensa concorrência e os produtos e serviços são semelhantes (Federação Brasileira de Bancos [FEBRABAN], 2008).

A estrutura de valor para o cliente tem justamente a finalidade de apontar quais atributos são mais relevantes para cada tipo de cliente, possibilitando a formulação de estratégias de sucesso e construção de uma base de informações necessárias para se projetar o valor financeiro do cliente durante a sua vida útil de consumo - customer lifetime value [CLV] (Rust, Zeithaml, \& Lemon, 2001). Além disso, pelo fato de a estrutura de valor para o cliente também permitir avaliações individualizadas e por segmentos de clientes, demonstra ser um instrumento útil aos bancos de varejo, por possibilitar ganhos de escala sem perder foco, em função da ampla e diversificada base de clientes que possuem (FEBRABAN, 2008).

Apesar da sua importância e abrangência, esse é um tema recente que ainda carece de pesquisas que considerem os fatores que influenciam os diversos tipos de clientes em diferentes contextos. Há indicadores de que modelos e escalas que vêm sendo desenvolvidos revelam ser incompletos, não contemplando todas as dimensões que identificam a percepção de valor pelo cliente, com escopo muito abrangente e, desse modo, imprecisos para uma operacionalização do CLV, como se verifica em Rust et al. (2001) e Parasuraman, Zeithaml e Berry (1988). Além disso, não vem sendo testada, de forma suficiente, a sua aplicabilidade e precisão junto a diversos setores e públicos-alvos, como bancos de varejo e seus diversos segmentos de cliente, embora haja amplo reconhecimento da sua utilidade para as organizações (Blattberg \& Thomas, 2001; Kotler, 2001; Reinartz \& Kumar, 2003; Rust et al., 2001).

Os bancos têm sido pioneiros na adoção de estratégias para conhecer melhor o cliente por meio dos seus padrões de comportamento de consumo e de atribuição de valor para, com isso, criar situações propícias para incrementar negócios. Apesar de já terem avançado bastante nesse campo, os bancos de varejo no Brasil ainda estão numa fase inicial de estabelecimento de formas de relacionamento com clientes, estruturadas principalmente em modelos de segmentação que se baseiam em parâmetros diversos, podendo variar de formas mais simples como níveis de renda, até outras mais sofisticadas, como a comportamental, que indicam hábitos e propensão de consumo. Contudo esse setor é reconhecido como um dos mais avançados, em termos de foco no cliente e de adoção de estratégias para estabelecer relações duradouras de consumo.

Assim, o objetivo desta pesquisa foi desenvolver uma escala para identificar e mensurar o nível de importância de atributos de valor para o cliente do setor bancário brasileiro de varejo, com vistas a aprimorar e ampliar a prestação de serviços aos seus clientes, com a contrapartida de uma relação negocial duradoura e rentável. Nesse sentido, buscou-se uma metodologia robusta, em termos de 
processos e testes qualitativos e quantitativos, adequada não só ao presente estudo como a outros com necessidades semelhantes.

\section{O CONCEITO DE VALOR}

O customer value, cuja tradução mais usual para a língua portuguesa é valor para o cliente, é entendido por Kotler (2000) e Rust et al. (2001) como a percepção do cliente quanto ao resultado obtido na compra e uso de um produto ou serviço na perspectiva de uma relação custo-benefício. Customer equity - valor do cliente, por sua vez, é uma expressão cunhada por Blattberg e Deighton (1996), ao proporem um modelo cuja base de sucesso é a atração e manutenção dos clientes considerados mais valiosos. Representa o valor presente líquido [VPL] das receitas proporcionadas por um cliente, apurado com base na estimativa do total dos seus valores de compra de produtos e serviços, ao longo de toda a sua vida útil de consumo numa mesma empresa, o que pode ser representado por um fluxo de caixa líquido descontado com base em margens de contribuição, tendo uma conotação de importância financeira do cliente para uma empresa (Blattberg \& Thomas, 2001; Rust et al., 2001). Além do customer value e do customer equity, um terceiro conceito se apresenta nesse contexto de marketing: o customer lifetime value [CLV] ou valor do cliente por toda a vida ou que para Rust et al. (2001) representa a operacionalização matemática do conceito de valor do customer equity.

Já a estrutura do valor para o cliente, um termo criado por Rust et al. (2001), tem como função refletir aquilo que o cliente entende, no momento presente, como valioso para o início e manutenção de um relacionamento leal e duradouro com uma organização, o que propicia um gerenciamento eficaz no sentido de incrementar o valor do cliente, no decorrer da sua vida útil de consumo. De acordo com essa perspectiva, a empresa passa a focar não só os negócios atuais com o cliente como também os futuros, adotando ações no presente que levem a um retorno consistente no longo prazo - princípio para a apuração do CLV. Dessa forma, a identificação adequada dos aspectos que cada cliente valoriza em uma empresa, ou seja, a estrutura de valor para o cliente é uma etapa necessária para uma gestão estratégica eficaz, a fim de ampliar o valor financeiro representado por um relacionamento negocial de longo prazo, conforme a Figura 1.

\section{Figura 1: Da Estrutura de Valor para o Cliente à Estrutura de Valor do Cliente}
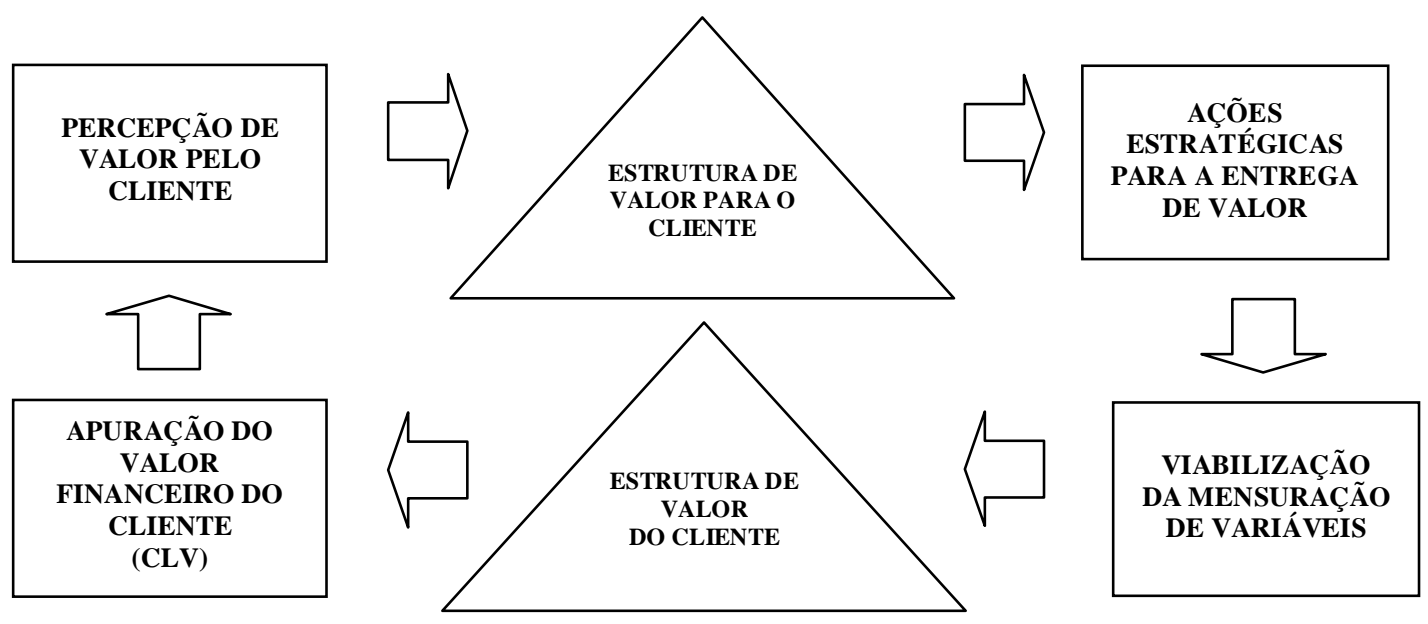

Fonte: elaborada pelo autor.

É nesse contexto que se situa o foco deste trabalho, considerando que a eficácia do processo depende do conhecimento prévio de como as organizações, neste caso, bancos de varejo, devem atuar no 
sentido de satisfazer aos interesses da sua clientela. De acordo com esse raciocínio, o valor do cliente seria de pouca utilidade, diante da ausência de informações a respeito da probabilidade de o cliente continuar a consumir os produtos e serviços do seu banco; ou até que ponto ele é leal, isto é, até quando ele está disposto a comprar, com exclusividade, de determinado banco. Ao adotar a perspectiva da estrutura de valor para o cliente, uma empresa assume efetivamente a posição e a postura de um fornecedor de produtos e serviços que busca conhecer o que o mercado consumidor deseja, para que com ele estabeleça uma relação de lealdade e com baixo risco de mudança de marca.

\section{Base Teórica da PercepÇÃo de VAlor}

Um dos primeiros pesquisadores a procurar sistematizar o conceito de valor no escopo da mercadologia foi Kotler (1972), quando, com vistas a desenvolver, de forma mais rigorosa, um conceito genérico de marketing e reforçar as bases das trocas sociais, apresentou quatro axiomas, com os respectivos corolários, destacando-se o quarto deles (Tabela 1):

\section{Tabela 1: O Quarto Axioma do Marketing}

\begin{tabular}{l|l}
\hline Axioma 4 & $\begin{array}{l}\text { Marketing é a tentativa de produzir a resposta desejada por meio da criação e oferta de } \\
\text { valores para o mercado. }\end{array}$ \\
\hline Corolário 4.1 & O mercador assume que a resposta do mercado será voluntária. \\
Corolário 4.2 & $\begin{array}{l}\text { A atividade essencial do mercado é a criação e oferta de valor. O valor é definido } \\
\text { subjetivamente sob o ponto de vista do mercado. }\end{array}$ \\
Corolário 4.3 & $\begin{array}{l}\text { O mercador cria e oferece valor, principalmente por meio da configuração, valorização, } \\
\text { simbolização e facilitação [Configuração é o ato de formatar o objeto social. A } \\
\text { valorização diz respeito ao estabelecimento dos termos de troca relativos ao objeto. }\end{array}$ \\
& $\begin{array}{l}\text { Simbolização é associação de significados sobre o objeto. Facilitação consiste na } \\
\text { alteração da acessibilidade ao objeto]. } \\
\text { "Marketing efetivo" significa as escolhas do mercador que são calculadas para produzir a } \\
\text { resposta desejada no mercado. "Marketing eficiente" significa a escolha das ações do } \\
\text { mercador de menor custo que irão produzir a resposta desejada. }\end{array}$ \\
\hline
\end{tabular}

Fonte: Kotler (1972, pp. 49-50).

A literatura de marketing aponta diversos construtos vinculados a valor, como antecedentes ou conseqüentes: preço, conveniência, qualidade, marca, relacionamento, retenção, confiança, satisfação, lealdade. Alguns estudos adotam uma perspectiva holística em torno do conceito de valor para o cliente, buscando mapear as camadas abstracionais subjacentes a esse construto. Dois modelos vêm recebendo maior atenção na comunidade acadêmica. Um deles é o de valor do cliente, de Rust et al. (2001), segundo o qual o consumidor é atraído pelo (1) valor da marca - conjunto de percepções simbólicas formadas em sua mente; (2) valor do valor - fatores que ele consegue, de alguma forma, mensurar e tangibilizar em termos econômicos; e (3) o valor de retenção, que é a concessão de compensações ao cliente por sua lealdade à empresa. O outro é o modelo SERVQUAL, de Parasuraman et al. $(1985,1988)$, que se vale de escala específica para avaliação de qualidade em serviços.

O SERVQUAL vem inspirando diversos estudos em todo o mundo, desde a sua divulgação. Entretanto também vem suscitando discussões teóricas e operacionais e sendo motivo de críticas, principalmente por aspectos metodológicos, com destaque para Babakus e Boller (1992); Bahia e Nantel (2000); Buttle (1996); Carman (1990); Lewis (1993). Um reparo comum a esses autores sobre o SERVQUAL diz respeito à dimensionalidade da sua escala, cujos subconstrutos são considerados 
insuficientes para abranger o composto de marketing e as peculiaridades dos diversos setores de serviços diante dos diferentes tipos de consumidores e ambientes físicos.

O modelo baseado no conceito de customer equity de Rust et al. (2001) reúne várias dimensões existentes na literatura sobre valor. Entretanto duas observações podem ser feitas quanto a empecilhos para que ele possa atingir o seu propósito, que é calcular o valor presente líquido (VPL) do cliente para uma organização, considerando uma relação duradoura de consumo: (1) a carência de variáveis relevantes para explicar determinado construto e até mesmo a ausência de construtos, o que pode levar a resultados imprecisos quanto a alguns mercados, como o de bancos de varejo; e (2) a operacionalização na identificação dos indicadores e construtos considerados como os mais importantes para o cliente, de forma segmentada ou individualizada.

Dessa forma, esses dois modelos podem ser úteis em termos de orientação teórica e mercadológica; mas, como foi exposto, fica evidente que não são inteiramente adequados para identificar os atributos de valor para clientes de bancos de varejo, principalmente pela falta de validade da sua escala para o estudo em foco. A identificação dos indicadores e respectivas dimensões está no âmago da operacionalização, que permite um modus operandi de ser replicado por outros pesquisadores (Kerlinger, 1979; Maxwell, 1996). Acrescente-se que tais estudos foram desenvolvidos e testados em contextos diferentes do brasileiro e, de acordo com a abordagem fenomenológica, especificidades locais devem ser consideradas, pois delas podem emergir subconstrutos diferentes, revelando uma situação distinta da que poderia ser verificada por meio da seleção de indicadores das dimensões aqui descritas. Portanto, a partir do conceito de formação da estrutura de valor para o cliente, tendo o SERVQUAL como modelo comparativo e os construtos identificados na literatura como referência teórica, foi desenvolvida uma escala própria, atendendo aos pressupostos de qualidade psicométrica: unidimensionalidade, confiabilidade, validade e normas, para aplicação junto a diferentes amostras (Bruner, 2003; Spector, 1992), e adequada à realidade brasileira.

\section{METODOLOGIA}

O desenvolvimento de uma escala específica, de forma a conferir maior precisão aos resultados, encontra amparo em Bruner (2003) que, de forma aparentemente paradoxal, condena a proliferação de escalas sob o argumento de que muitas delas levam às mesmas conclusões, quando são utilizadas em pesquisas empíricas, gerando desperdício de recursos. A essência da sua crítica repousa na falta de explicação pelos pesquisadores das razões para desenvolverem nova escala nos dias atuais, em que o acesso às informações científicas se tornou bastante facilitado com o advento da Internet.

Contudo ressalta que essa decisão é cabível, quando não houver escala disponível ou, quando existir, seja inadequada de alguma forma, seja pela falta de validade aparente ou especificidades do novo estudo, como é o presente caso, como foi exposto. Além disso, o escopo do trabalho perpassa, necessariamente, pela reunião de métodos que propiciem uma metodologia robusta para o desenvolvimento de escalas com maior validade e confiabilidade.

O desenvolvimento da escala teve como suporte teórico autores considerados referências na área (Churchill, 1979; Jacoby, 1978; Mathieson, 1988; Maxwell, 1996; Miles \& Huberman, 1994; Selltiz, Wrightsman, \& Cook, 1987; Spector, 1988, 1992). Devido às características da situação-problema, a triangulação teve papel relevante no estudo, em que diversas técnicas e testes qualitativos precederam a fase de análises quantitativas. A aplicação de diversos testes também foi relevante; os de caráter qualitativo aplicados na validação da escala, assim como as providências adotadas, estão descritos na Tabela 2. 
Tabela 2: Testes Qualitativos de Validade

\begin{tabular}{|c|c|c|}
\hline Teste & Descrição & Providências adotadas \\
\hline $\begin{array}{l}\text { Abordagem modus } \\
\text { operandi }\end{array}$ & $\begin{array}{l}\text { Método de investigação e descrição de eventos, } \\
\text { assemelhado a uma listagem dos principais passos } \\
\text { de um processo e das ameaças à validação. }\end{array}$ & $\begin{array}{l}\text { Relatório sucinto de todos os } \\
\text { passos da pesquisa. }\end{array}$ \\
\hline $\begin{array}{l}\text { Busca por evidências } \\
\text { discrepantes e casos } \\
\text { negativos }\end{array}$ & $\begin{array}{l}\text { Identificação e análise de dados e casos que possam } \\
\text { tornar falsa uma possível conclusão. }\end{array}$ & $\begin{array}{l}\text { Busca de detalhamento de } \\
\text { depoimentos e na análise de } \\
\text { questionários preenchidos. }\end{array}$ \\
\hline Triangulação & $\begin{array}{l}\text { Coleta de informações de várias fontes por meio de } \\
\text { diversos métodos. }\end{array}$ & $\begin{array}{l}\text { Grupos de foco, entrevistas } \\
\text { pessoais, painéis de } \\
\text { especialistas, feedbacks. }\end{array}$ \\
\hline Feedback & $\begin{array}{l}\text { Solicitação da opinião de outras pessoas para } \\
\text { identificar ameaças à validade, os vieses do próprio } \\
\text { pesquisador e as imperfeições na lógica, métodos e } \\
\text { técnicas da pesquisa. }\end{array}$ & $\begin{array}{l}\text { Junto a clientes de bancos e } \\
\text { especialistas acadêmicos e de } \\
\text { bancos. }\end{array}$ \\
\hline Dados 'ricos' & $\begin{array}{l}\text { Transcrição literal de entrevistas, em vez de simples } \\
\text { notas, cujo detalhamento proporcione um quadro } \\
\text { real do evento que está ocorrendo. }\end{array}$ & $\begin{array}{l}\text { Todas as entrevistas foram } \\
\text { transcritas pelos próprios } \\
\text { pesquisadores. }\end{array}$ \\
\hline Quase-estatísticas & $\begin{array}{l}\text { Referem-se ao uso de resultados numéricos simples } \\
\text { que podem ser prontamente deduzidos dos dados. }\end{array}$ & $\begin{array}{l}\text { Incluídas no relatório de } \\
\text { pesquisa. }\end{array}$ \\
\hline Comparação & $\begin{array}{l}\text { Confronto dos resultados obtidos em diferentes } \\
\text { situações ou segmentos do público pesquisado, a } \\
\text { fim de detectar dados divergentes ou que ratifiquem } \\
\text { os resultados. }\end{array}$ & $\begin{array}{l}\text { Comparação a partir dos } \\
\text { depoimentos dos diferentes } \\
\text { segmentos de clientes. }\end{array}$ \\
\hline $\begin{array}{l}\text { Validade de face ou de } \\
\text { conteúdo }\end{array}$ & $\begin{array}{l}\text { Avaliações subjetivas, porém sistemáticas do nível } \\
\text { de exatidão com que o conteúdo da escala é capaz } \\
\text { de fazer a medição pretendida. A avaliação pode ser } \\
\text { feita pelo pesquisador ou por outras pessoas, por } \\
\text { meio de painéis com especialistas. }\end{array}$ & $\begin{array}{l}\text { Três painéis de especialistas } \\
\text { com: }\left(1^{\circ}\right) \text { três professores } \\
\text { doutores; }\left(2^{\circ}\right) \text { cinco } \\
\text { mestrandos; }\left(3^{\circ}\right) \text { seis } \\
\text { especialistas de bancos. }\end{array}$ \\
\hline $\begin{array}{l}\text { Pré-teste dos } \\
\text { questionários }\end{array}$ & $\begin{array}{l}\text { Busca identificar e eliminar problemas potenciais, } \\
\text { devendo ser feito, preferencialmente, por meio de } \\
\text { entrevistas pessoais. }\end{array}$ & $\begin{array}{l}\text { Dois pré-testes: }\left(1^{\circ}\right) 20 \\
\text { questionários; }\left(2^{\circ}\right) 21 \\
\text { questionários; com obtenção } \\
\text { de feedbacks em } 31 \text { casos. }\end{array}$ \\
\hline Member check & $\begin{array}{l}\text { Submissão do instrumento a especialistas } \\
\text { acadêmicos e de mercado para coleta de feedbacks } \\
\text { e submissão dessas análises a todos eles, } \\
\text { preservando o anonimato, de forma a confirmar ou } \\
\text { alterar as avaliações do pesquisador. }\end{array}$ & $\begin{array}{l}\text { Questionário submetido a } \\
\text { duas doutorandas e três } \\
\text { mestrandos com intervalo de } \\
\text { duas semanas. }\end{array}$ \\
\hline
\end{tabular}

Fonte: compilado pelo autor.

De acordo com Churchill (1979), o processo de desenvolvimento de uma escala se desenvolve em oito etapas.

\section{Primeira Etapa: Identificar o Domínio Específico do Construto}

Nesta etapa, o objetivo é conceitualizar o construto e dimensionar o seu domínio, buscando-se razões e evidências para justificar a necessidade de nova escala de mensuração (Bruner, 2003). Por meio da análise de modelos e escalas disponíveis na literatura, chegou-se à conclusão quanto à necessidade de um instrumento capaz de identificar dimensões de valor e seus indicadores, bem como o nível relativo de importância de cada um deles, por parte de clientes de bancos de varejo no Brasil, por segmento. 


\section{Segunda Etapa: Gerar uma Amostra de Itens}

Esta etapa, de caráter exploratório, destina-se a gerar itens que expliquem o domínio do construto especificado. Foi realizada ampla pesquisa bibliográfica, seguida de entrevistas por meio de minigrupos de foco - forma mais adequada, quando o assunto exige investigação extensa e aprofundada (Malhotra, 2001). Buscou-se uma amostra representativa dos clientes de bancos de varejo no Brasil - arcabouço amostral. Devido à elevada quantidade de componentes desse contingente, buscou-se selecionar depoentes de forma não probabilística, por conveniência, reunindo pessoas com diferentes níveis de renda, sendo este geralmente o primeiro e principal critério de segmentação utilizado pelos bancos. Além disso, foram aplicados critérios de seleção, como idade, gênero, estado civil, escolaridade e ocupação. O limitador para a quantidade de entrevistas seria quando os depoimentos se tornassem repetitivos. Dessa forma, foram reunidas 38 pessoas em oito minigrupos de foco heterogêneo, conforme aplicado por Corfman (1995); a quantidade de componentes de cada grupo variou de quatro a seis. Nas entrevistas, conduzidas pelo pesquisador, com tempo aproximado de uma hora cada, a pergunta inicial para cada respondente referiu-se aos fatores que ele considerava como os mais importantes no momento atual da sua vida para que um banco fosse considerado o banco ideal, de forma que a ele se mantivesse leal, em perspectiva de longo prazo. Também foram exploradas dimensões identificadas na literatura, sendo a maioria dos depoimentos baseada em incidentes críticos, o que conferiu maior confiabilidade aos relatos. Após cada sessão de minigrupo de foco, as declarações foram transcritas e analisadas por meio de análises de conteúdo e semântica, sendo identificadas 117 variáveis. Em seguida, foram promovidas novas análises qualitativas, feedbacks de entrevistados e de especialistas acadêmicos e de bancos, análise semântica e análise de conteúdo que reduziram as variáveis a 91.

Em seguida passou-se à identificação dos subconstrutos, a partir das variáveis remanescentes, valendo-se das categorizações contidas em Parasuraman et al. (1988). Além disso, após um processo de member check e rodadas de feedbacks com respondentes e especialistas acadêmicos e de bancos de varejo, chegou-se a um número final de 82 variáveis, agrupadas em 10 subconstrutos assim identificados: comunicação, imagem da marca, confiabilidade, custo-benefício, conveniência, prontidão da resposta, personalização, postura funcional, benevolência e instalações físicas.

$\mathrm{Na}$ seqüência, foram definidos os formatos da escala e do questionário, com sucessivos aprimoramentos até chegar ao modelo definitivo, cuja validade foi obtida por meio dos testes descritos na Tabela 2. A forma da escala foi definida como multiitem do tipo Likert, equilibrada com seis pontos, mas não forçada, com inclusão de alternativa para casos em que o respondente não tem opinião formada. Quanto ao questionário, dedicou-se especial atenção às instruções iniciais para evitar os efeitos indesejáveis provocados pelos chamados social desirability e aquiescence response set (Spector, 1992), principalmente em função da sua extensão - 82 itens, sendo 17 com sentido inverso. A escala foi denominada de Valor no Varejo Bancário Brasileiro [VVBB] Matriz - por servir de matriz para escalas destinadas a segmentos específicos de clientes, como será tratado adiante.

\section{Terceira Etapa: Coleta de Dados}

A validação qualitativa da escala VVBB-Matriz a credenciou a ser aplicada junto ao conjunto de clientes de bancos de varejo. Contudo, para obter maior precisão nas informações e elaborar estratégias empresariais com maiores chances de sucesso, é recomendável desenvolver uma escala direcionada ao segmento-alvo. Nesse escopo, a partir da escala VVBB-Matriz, foi desenvolvida nova escala, direcionada ao segmento Alta Renda, geralmente composto por clientes bancários com renda bruta acima de $\mathrm{R} \$ 10$ mil mensais e/ou saldo de aplicações financeiras acima de $\mathrm{R} \$ 50$ mil. Esse processo requer coleta de dados mediante um survey, com vistas a proceder a análises estatísticas. Os dados foram coletados junto à amostra de clientes Alta Renda de um grande banco de varejo instalado no país, doravante chamado de Banco. Dessa forma, a partir do refinamento da escala VVBB-Matriz, buscou-se desenvolver a escala VVBB-Alta Renda. 
A escala VVBB-Matriz foi aplicada na região metropolitana de Belo Horizonte (MG). O arcabouço amostral, ou seja, a lista ou conjunto de instruções para identificar a população-alvo, foi providenciado pelo Banco, que realizou um levantamento da quantidade dos seus clientes que fazem parte desse público-alvo na referida região. A unidade amostral foi o conjunto das agências do Banco selecionadas para a coleta de dados junto aos seus clientes. Das 39 agências identificadas foram selecionadas 16, por proporcionarem melhores condições operacionais para a coleta de dados, sem prejuízo da distribuição espacial homogênea das amostras, pois estão geograficamente localizadas em toda a região metropolitana de Belo Horizonte (MG). Como essas 16 dependências concentram a maior parte dos clientes componentes do público-alvo, elevou-se a possibilidade de encontrar o cliente no ambiente físico de atendimento e assim proceder à coleta de dados. A amostragem foi não probabilística e por conveniência, sendo respondidos 270 questionários, reduzidos a 227, após as análises qualitativas e quantitativas.

Hair, Anderson, Tatham e Black (1998) não recomendam análises multivariadas com um número de casos inferior a cinco por variável. No entanto Spector (1992) considera como satisfatória uma quantidade de 100 a 200 questionários, parâmetro utilizado por diversos pesquisadores de renome, em estudos para desenvolvimento de escalas destinadas a analisar a satisfação com o trabalho (Churchill, Ford, \& Walker, 1974), qualidade em serviços (Parasuraman et al., 1985, 1988) e orientação para o cliente por parte de profissionais de vendas (Saxe \& Weitz, 1982).

\section{Quarta Etapa: Purificação da Medida}

Nesta etapa, inicialmente, deve ser feita a preparação dos dados para as análises estatísticas por meio de processos qualitativos e quantitativos. Em seguida é realizada uma análise fatorial exploratória, para detectar novos subconstrutos e respectivas variáveis, de acordo com as percepções do públicoalvo. Os resultados devem então ser submetidos a análises de confiabilidade e validades convergente e discriminante.

\section{Identificação de Evidências Discrepantes e Casos Negativos}

Foram detectados nove casos com ausência de respostas superior a $10 \%$ dos itens e três casos com marcações N (Não tenho opinião formada), acima de $15 \%$.

\section{Edição e Codificação}

Foram excluídos 25 casos, por não observação dos itens inversos, marcações repetidas em seqüência, assinalamentos indicando displicência do respondente e aparente incompatibilidade entre idade e renda.

\section{Análise Gráfica das Variáveis}

A análise dos histogramas das variáveis não permitiu nenhuma conclusão quanto à normalidade, o que foi avaliado por meio de teste estatístico apropriado, como recomendam Hair et al. (1998), descrito adiante.

\section{Análise de Missing Values}

Para Malhotra (2001), a tolerância para os dados ausentes se limita a 10\% do total dos itens do questionário, o que gerou a exclusão de três casos. Para os demais dados ausentes foi feita a imputação de um valor neutro, pela média da variável, um dos métodos mais largamente usados por não afetar outras análises estatísticas, como a correlação, porque o valor médio da variável permanece inalterado (Hair et al., 1998). 


\section{Análise de Outliers}

Realizada por meio de análise multivariada, com a divisão da Distância de Mahalanobis $\left(D^{2}\right)$, que é a medida da distância entre o ponto médio central do conjunto formado pelas observações e a posição de cada uma no espaço multidimensional, pelos graus de liberdade $(g l)$. O resultado tem de ser superior ao nível de significância de 0,001 - limite para a indicação de um outlier (Hair et al., 1998). É interessante destacar que esses autores se limitaram a atribuir o nível do teste, não indicando, porém, um valor para se comparar com o resultado da referida equação. Neste caso, utilizou-se como referência o valor de 3,160, correspondente ao mesmo nível de significância $(\alpha=0,001)$ para $120 \mathrm{gl}$, conforme a tabela das distribuições $t$ de Student. Portanto, considerando $g l=82$, para qualquer valor abaixo de 3,232 assumiu-se a hipótese nula de que não se trata de outlier. Como o maior valor apurado foi 1,163 , nenhum questionário foi retirado por esse critério.

A análise bivariada considera pares de variáveis por meio de um gráfico de dispersão, ou scatterplot. Com 82 variáveis, são possíveis 3.321 combinações e a análise de cada uma delas mostrou-se inviável, razão pela qual não foi realizada. Na análise univariada foram identificadas as observações cujas médias se afastavam mais que três desvios-padrão da média do conjunto de dados, quando foram encontrados vários casos de outliers. Entretanto, devido ao propósito do trabalho de obter percepções pessoais sobre valor, toda resposta entre as previstas foi considerada aceitável, razão por que esse critério não determinou exclusão de informações. Como ressaltaram Hair et al. (1998), "o pesquisador deve abster-se de classificar muitas observações como outliers, não sucumbindo à tentação de eliminar os casos não-consistentes com os remanescentes somente porque eles são diferentes" (p. 66). Apesar disso, a partir das indicações desse teste, foi feita nova análise qualitativa dos questionários, que indicou seis casos com falta de coerência nas respostas, principalmente nas questões reversas. Após essas análises preparatórias, o saldo de questionários válidos ficou sendo 227.

\section{Análise de Normalidade}

Foi realizada por meio do cálculo de valores para a estatística $z$, para a assimetria e para a curtose. Os resultados dos testes de normalidade univariada revelaram que nenhuma das variáveis segue a distribuição normal. Hair et al. (1998) recomendam que se tente aplicar métodos de transformação dos dados para normalizar os escores, o que foi feito por meio da forma inversa $(1 / x)$ e pela raiz quadrada, com a aplicação de logaritmos, sem sucesso. Contudo a premissa de normalidade dificilmente é satisfeita em estudos na área de ciências sociais. Johnson e Wichern (1998), assim como West, Finch e Curran (1995) reconheceram a dificuldade de obter, no mundo real, dados que se comportem de forma a seguir rigorosamente uma distribuição normal. Ruekert e Churchill (1984), diante da mesma situação, ao desenvolverem uma escala, deram continuidade às análises, recomendando, porém, cautela quanto aos resultados. Reforçando que a precisão matemática não deve ser um impeditivo para os estudos sobre o mundo da observação, Guilford $(1959$, p. 6) afirmou:

A antiga idéia dos gregos de que o mundo funciona numa base matemática é incorreta. Matemática é uma invenção do homem, não uma descoberta. .... A natureza nunca é explicada exatamente por qualquer modelo matemático. Todas as descrições são apenas aproximações, algumas melhores, outras piores.

\section{Análise de Linearidade}

Foi realizada com a avaliação da significância do coeficiente de correlação produto-momento de Pearson $(r)$ para as variáveis de um mesmo construto. Qualquer valor diferente de zero e sendo significativo confirma a existência de uma correlação linear. As correlações entre cada item com o total dos subconstrutos também são indicadores robustos e relevantes para o desenvolvimento de escalas (Gerbing \& Anderson, 1988; Nunnally \& Bernstein, 1994). Os resultados indicaram falta de correlação significativa no nível de $1 \%$ entre diversas variáveis, indicando a necessidade de proceder a uma análise fatorial exploratória (AFE), para detectar os construtos subjacentes às percepções dos respondentes, em público de Alta Renda. 


\section{Análise de Confiabilidade}

Foi feita por meio de teste-reteste e apuração do alfa de Cronbach para cada subconstruto. Apesar das controvérsias teóricas sobre a confiabilidade do teste-reteste, em função da memória dos respondentes (Churchill, 1979; Guilford, 1959; Malhotra, 2001), procedeu-se a esse tipo de teste junto a um público formado por 15 clientes de bancos de varejo, aos quais foi submetido o questionário de pesquisa, com intervalo de tempo entre o teste e o reteste em torno de 30 dias; a comparação das médias não indicou diferenças significativas em termos estatísticos em $89 \%$ dos itens. Em seguida foi realizado o teste de confiabilidade interna, por meio do cálculo do alfa de Cronbach, de cada subconstruto da escala VVBB-Matriz, com exclusão de 21 variáveis que contribuíam para a redução da consistência interna. Quatro subconstrutos apresentaram alfas abaixo de 0,6 e dois acima de 0,7. Malhotra (2001) admite alfas a partir de 0,6, enquanto Hair et al. (1998) só concordam com escores abaixo de 0,7 em pesquisas exploratórias, como no presente caso. Cumpre considerar que os subconstrutos iniciais foram definidos a partir de dados coletados no mundo real, analisados por meio de técnicas qualitativas e referendadas por especialistas acadêmicos. Além disso, a amostra da escala VVBB-Matriz foi composta por um contingente mais amplo de perfis de cliente e depois aplicada a um segmento específico. Em situações como essa, é natural e até esperada a emergência de construtos que representem a percepção de valor desse público, o que se coaduna com os propósitos deste estudo.

\section{Análise Fatorial Exploratória [AFE]}

Inicialmente, foi necessário avaliar se a amostra era adequada para o uso de AFE, valendo-se do cálculo do índice de Kaiser-Meyer-Olkin [KMO], que indica se a correlação entre os pares de variáveis pode ser explicada por outras variáveis. Os resultados apurados para todos os subconstrutos se situaram entre 0,5 e 1,0, habilitando a aplicação da AFE. O outro teste é o de esfericidade de Bartlett, que verifica em que medida há correlação entre as variáveis; um valor com significância menor que 0,05 recomenda o uso da análise fatorial, o que foi constatado para todos os subconstrutos.

A AFE foi realizada com os dez subconstrutos da escala VVBB-Matriz e as respectivas 61 variáveis remanescentes. Para extração dos fatores, foi utilizada a técnica de análise de componentes principais, recomendada por Spector (1992), tendo em vista o objetivo de explorar a dimensionalidade da escala. Além disso, é a mais indicada em situações de não-detecção de normalidade na distribuição das variáveis (Guilford, 1959; Ruekert \& Churchill, 1984). Foi utilizado o método de rotação oblíqua da matriz de fatores para torná-la mais fácil de ser interpretada, em função da natural ocorrência de variáveis com cargas significativas em mais de um fator. A matriz fatorial foi interpretada por meio das cargas fatoriais, a partir do nível mínimo recomendado por Hair et al. (1998), que é de $\pm 0,30$. Foram buscados níveis mais elevados com a meta de atingir $\pm 0,50$, patamar considerado praticamente significante. Para Bagozzi (1984), \pm 0,50 é o piso tolerável. Foram feitas quatro rodadas de análises, com a exclusão de variáveis com cargas ambíguas - assim consideradas aquelas com diferenças inferiores a 0,100 nas cargas dos fatores - até que, restasse somente um fator com autovalor acima de um por dimensão. Ao final emergiram cinco novos subconstrutos, com um total de 32 variáveis (vide APÊNDICE).

\section{Análise de Unidimensionalidade de Construto}

Optou-se pela AFE para analisar a unidimensionalidade por ser a mais usual e acessível a este tipo de estudo. Toda dimensão que apresentar somente um fator, com autovalor maior que um, será considerada unidimensional. Detectou-se que o subconstruto Empatia se revelou quadridimensional e o subconstuto Confiabilidade bidimensional, enquanto os demais, unidimensionais. A exclusão de quatro variáveis que apresentaram cargas ambíguas solucionou a deficiência nos subconstrutos Empatia e Confiabilidade. Foi calculado o alfa de Cronbach para os novos subconstrutos. Benevolência e Competência, com alfas 0,54, apresentaram o menor número de variáveis, três e quatro, respectivamente, o que pode ter afetado esse resultado. Considerando o caráter exploratório do 
estudo, esses subconstrutos não foram excluídos com vistas a observar seu comportamento diante das análises de validade. Os valores dos demais alfas foram a partir de 0,70.

\section{Análise de Validade Convergente}

Para Churchill (1979), uma forma útil de obter a validade convergente é por meio da matriz multitraços-multimétodos (MMTX). Contudo esse método é apontado por Bagozzi, Yi e Phillips (1991) como insuficiente para disponibilizar resultados confiáveis, sugerindo a análise fatorial confirmatória (AFC), em que a covariância entre os construtos é avaliada. Dessa forma, são consideradas convergentes as variáveis com cargas superiores a 0,500 e valor $\mathrm{p}<0,05$ (Dunn, Seaker, $\&$ Waller, 1994). Outra técnica é apurar, entre os itens de um mesmo construto, se os resultados do valor de $t$ são maiores que 1,96 - valor crítico para o nível de significância de 0,05 - para demonstrar que a carga de cada indicador no subconstruto é significativa em termos estatísticos (Bagozzi, 1981; Bagozzi et al., 1991). Foi constatado que todas as variáveis convergem dentro dos respectivos subconstrutos, o que significa que elas se correlacionam para medir a mesma coisa.

\section{Análise de Validade Discriminante}

A validade discriminante foi averiguada pelo teste de diferença de qui-quadrados $\left(\chi^{2}\right)$, comparandose os subconstrutos em pares por meio de dois modelos: o primeiro com covariância igual a um e o segundo com covariância livre. A validade discriminante foi constatada pela redução no valor da estatística qui-quadrado do segundo modelo em relação ao valor do primeiro, cuja diferença se mostrou superior a 3,84, assegurando que os construtos medem coisas diferentes (Moorman \& Miner, 1998).

\section{Quinta Etapa: Desenvolvimento de Normas}

As normas definem como classificar e avaliar o grau de concordância do respondente com a importância do conjunto de valores previstos na escala, que varia de acordo com a quantidade de itens e a soma dos escores assinalados: de zero, em função da opção N, até 192 (32 variáveis x 6). Foi efetuada uma distribuição de freqüência e estabelecidos os intervalos mínimos e máximos para que não houvesse lacunas entre as classes de escore, com notas variando de zero, pior nível de concordância, a dez, melhor nível de concordância.

\section{Comparações com Explanação de Referência}

Miles e Huberman (1994) afirmam que levar em conta outras formas de explicação para o mesmo fenômeno "que vem sendo cuidadosamente estudado e despoticamente esclarecido é um exercício saudável de autodisciplina e uma forma de evitar a arrogância resultante de orgulho ou paixão excessivos" (p. 274). Portanto, após a definição da escala, foi feita uma comparação das suas características com as de outro modelo de referência que busca mensurar fenômeno semelhante também chamado de modelo rival - avaliando as vantagens e desvantagens. O modelo selecionado foi o SERVQUAL, também utilizado para esse fim por Bahia e Nantel (2000). Os resultados apontam diferenças favoráveis à escala desenvolvida neste estudo, bem como algumas semelhanças com o SERVQUAL. 
Tabela 3: Comparativo Escala VVBB-Alta Renda versus SERVQUAL

\begin{tabular}{l|l}
\hline \multicolumn{1}{c|}{ VVBB-Alta Renda } & \multicolumn{1}{c}{ SERVQUAL } \\
\hline $\begin{array}{l}\text { Maior validade de conteúdo por destinar-se a um } \\
\text { contexto específico de serviços - bancos de varejo no } \\
\text { Brasil }\end{array}$ & $\begin{array}{l}\text { Busca abranger todos os contextos de serviços a } \\
\text { partir da realidade estadunidense }\end{array}$ \\
\hline Contempla a dimensão Preço & Ignora a dimensão Preço \\
\hline $\begin{array}{l}\text { Imagem da empresa desassociada da imagem do } \\
\text { funcionário (Empatia) }\end{array}$ & $\begin{array}{l}\text { Imagem da empresa associada à imagem do } \\
\text { funcionário (Responsiveness) }\end{array}$ \\
\hline Todas as cargas fatoriais acima de 0,500 & $\begin{array}{l}\text { Algumas cargas abaixo de 0,500 (Babakus \& } \\
\text { Boller, 1992) }\end{array}$ \\
\hline Submetida a teste de Validade Discriminante & $\begin{array}{l}\text { Não submetida a teste de Validade Discriminante } \\
\text { (Bahia \& Nantel, 2000) }\end{array}$ \\
\hline \multicolumn{2}{c}{ Itens semelhantes em dimensões diferentes (Empatia X Tangibles) } \\
\hline \multicolumn{2}{c}{ Dimensões semelhantes nos dois modelos: Confiabilidade/Reliability; Competência/Assurance }
\end{tabular}

Fonte: compilado pelo autor a partir dos dados da pesquisa.

\section{CONSIDERAÇÕES SOBRE O ESTUDO}

\section{Implicações Acadêmicas e Gerenciais}

A maior contribuição deste estudo, em termos teóricos e acadêmicos, pode ser creditada à operacionalização do construto valor para o cliente, possibilitando que ele seja verificado empiricamente em contexto particular. Como demonstra a Tabela 3, uma escala específica apresenta vantagens em relação ao uma já existente, mesmo com adaptações. A escala SERVQUAL, apesar de amplamente utilizada em todo o mundo, foi desenvolvida em contexto diferente. A partir deste estudo, o posicionamento de Bruner (2003) pode ser abordado sob duas perspectivas: (1) a da concordância, diante da sua afirmativa de que uma nova escala tem de ser justificada, e o da (2) discordância quanto ao combate à proliferação de escalas, propondo-se que se busque desenvolver instrumentos específicos, em vez de se valer do recurso da adaptação, desde que o contexto do problema de pesquisa o justifique. Dessa forma, a presente proposta, acompanhada da operacionalização para o desenvolvimento de escalas próprias para identificar atributos valorizados pelo consumidor, de forma segmentada, bem como para avaliar qualidade em serviços, representa um avanço nos campos da pesquisa e das estratégias empresariais.

À metodologia utilizada no trabalho pode ser atribuído nível similar de importância ao da própria escala. A utilização de preceitos de triangulação com a coleta de dados primários junto ao públicoalvo, cujos depoimentos espelham a vida real, compondo uma rica variedade de testes qualitativos, proporcionam consistência epistemológica ao estudo. A descrição de todos os passos para consecução da pesquisa implica a possibilidade de que o estudo não seja um fim em si mesmo, dada a possibilidade de ser replicado, aprimorado e testado pela comunidade científica.

Sob o aspecto gerencial, as organizações vêm demandando estudos mais precisos para buscar atender aos interesses dos seus clientes, visando à sua satisfação e lealdade, tendo em vista a lucratividade sustentável em longo prazo. Tal necessidade espelha a contribuição da estrutura de valor para o cliente, indicando, de forma clara, que ações devem ser priorizadas pelas organizações na elaboração das suas estratégias mercadológicas. O modelo forma a base para o cálculo do valor do cliente, o que permite estimar receitas futuras por meio do CLV, mesmo diante de um ambiente cada vez mais competitivo. 
Por meio da estrutura de valor para o cliente, a empresa pode adotar medidas estratégicas capazes de provocar maior impacto, avaliá-las ao longo do tempo, compará-las com as dos concorrentes e ajustálas tempestivamente por meio da avaliação dos resultados e da contínua obtenção de informações junto ao cliente sobre a sua percepção atual de valor. Nesse propósito, a escala VVBB-Alta Renda é um instrumento capaz de orientar o Banco - para o qual foi especialmente desenvolvida - por meio da apuração de médias para variáveis e subconstrutos, bem como por intermédio de escores por cliente e segmento, conforme normatizado. Contudo, diante da dinâmica do mercado e alterações no comportamento do consumidor, a escala VVBB-Alta Renda deve ser objeto de permanente atualização, da mesma forma que a VVBB-Matriz e suas possíveis derivadas. O detalhamento da metodologia e do seu modus operandi permite que o processo possa ser refeito pelos pesquisadores, até mesmo em outros setores que não o bancário de varejo, com maior agilidade, precisão e redução de despesas, além da alocação de investimentos nas ações com maior potencial de geração de resultados positivos.

\section{Limitações do Trabalho e Indicações de Novas Pesquisas}

Os alfas de alguns construtos podem ser considerados baixos. O fato de Hair et al. (1998) admitirem tais resultados em estudos exploratórios, não exime a necessidade de novas análises. Para tanto devese selecionar uma amostra representativa do público Alta Renda do Banco, de forma aleatória, com vistas a melhores resultados, em termos de confiabilidade, já que Churchill (1979) atribuiu à amostragem a maior fonte de resultados deficientes. Esse autor sugeriu, ainda, o retorno à primeira $\mathrm{e}$ segunda etapas, revendo o processo, para verificar se ocorreu algo errado. Assim, o aprimoramento do método é capaz de tornar o modelo de estrutura de valor para o cliente e seus instrumentos de mensuração - as escalas - mais confiáveis e válidos. Especial atenção deve ser dispensada à redação dos itens para produzir sentido inverso, à vista da complexidade do tema valor, conforme recomenda Carman (1990). Neste estudo foi elevada a eliminação desse tipo de item nas diversas análises. Outros aspectos a serem considerados são a não satisfação da premissa de normalidade, o que recomenda cautela quanto aos resultados, como alertam Ruekert e Churchill (1984) e a necessidade de novos estudos empíricos para testar a validade da escala, junto a uma amostra que possa ser considerada estatisticamente significativa.

Finalmente, dos resultados surgem possibilidades de futuros estudos no sentido de: (1) identificar a cadeia nomológica dos subconstrutos que se relacionam com o construto valor para o cliente; (2) desenvolver escalas destinadas a outros segmentos do setor bancário e empresas do mercado de serviços; (3) elaborar estratégias para obter maior retorno de marketing a partir do modelo em foco; e (4) avaliar o comportamento do consumidor em relação à efetividade da oferta de valor pelas organizações, no sentido de promover lealdade duradoura nas relações de consumo.

Artigo recebido em 25.06.2006. Aprovado em 25.06.2008.

\section{REFERENNCIAS BIBLIOGRÁFICAS}

Babakus, E., \& Boller, G. W. (1992). An empirical assessment of the SERVQUAL scale. Journal of Business Research, 24(3), 253-268.

Bagozzi, R. P. (1981). Evaluating structural equation models with unobservable variables and measurement error: a comment. Journal of Marketing Research, 18(3), 375-381.

Bagozzi, R. P. (1984). A prospectus for theory construction in marketing. Journal of Marketing, 48, 11-29. 
Bagozzi, R. P., Yi, Y., \& Phillips, L. W. (1991). Assessing construct validity in organizational research. Administrative Science Quarterly, 36(3), 421-458.

Bahia, K., \& Nantel, J. (2000). A reliable and valid measurement scale for perceived service quality of banks. International Journal of Bank Marketing, 18(2), 84-91.

Blattberg, R. C., \& Deighton, J. (1996). Manage marketing by the customer equity test. Harvard Business Review, 74(4), 136-144.

Blattberg, R. C., \& Thomas, J. S. (2001). Valuing, analyzing, and managing the marketing function using customers equity principles. In D. Iacobucci (Ed.). Kellog on marketing (pp. 302-329). New York: John Wiley \& Sons.

Bruner, G. C. II (2003). Combating scale proliferation. Journal of Targeting, Measurement and Analysis for Marketing, 11(4), 362-372.

Buttle, F. (1996). SERVQUAL: review, critique, research agenda. European Journal of Marketing, $30(1), 8-32$.

Carman, J. M. (1990). Consumer perceptions of service quality: an assessment of the SERVQUAL dimensions. Journal of Retailing, 66(1), 33-55.

Churchill, G. A., Jr. (1979). A Paradigm for developing better measures of marketing constructs. Journal of Marketing Research, 16, 64-73.

Churchill, G. A., Jr., Ford, N. M., \& Walker, O. C., Jr. (1974). Measuring the job satisfaction of industrial salesman. Journal of Marketing Research, 11(3), 254-260.

Corfman, K. P. (1995). The importance of member homogeneity to focus group quality. Advances in Consumer Research, 22(1), 354-359.

Dunn, S. C., Seaker, R. F., \& Waller, M. A. (1994). Latent variable in business logistics research: scale development and validation. Journal of Business Logistics, 15(2), 145-173.

Federação Brasileira de Bancos. (2008). O setor bancário em números. Recuperado em 17 março, 2008 , http://www.febraban.org.br/p5a_52gt34++5cv8_4466+ff145afbb52ffrtg33fe36455li5411pp+e/si tefebraban/informacoes_do_setor.pdf

Gerbing, D. W., \& Anderson, J. C. (1988). An updated paradigm for scale development incorporating unidimensionality and its assessment. Journal of Marketing Research, 25(2), 186-192.

Guilford, J. P. (1959). Psychometric methods (2nd ed.). Tokyio: Kogakusha.

Hair, J. F., Jr., Anderson, R. E., Tatham, R. L., \& Black, W. C. (1998). Multivariate data analysis (5th ed.). Upper Saddle River: Prentice Hall.

Jacoby, J. (1978). Consumer research: a state of the art review. Journal of Marketing, 42(2), 87-96.

Johnson, R. E., \& Wichern, D. W. (1998). Applied multivariate statistical analysis (4th ed.). New Jersey: Prentice-Hall.

Kerlinger, F. N. (1979). Metodologia da pesquisa em ciências sociais. São Paulo: EPU.

Kotler, P. (1972). A generic concept of marketing. Journal of Marketing, 36(2), 46-54.

Kotler, P. (2000). Administração de marketing: a edição do novo milênio. São Paulo: Prentice-Hall. 
Kotler, P. (2001). Reflections on marketing. In D. Iacobucci (Ed.). Kellog on marketing (pp. xiii-xvi). New York: John Wiley \& Sons.

Lewis, B. R. (1993). Service quality measurement. Marketing Intelligence and Planning, 11(4), 4-12.

Malhotra, N. K. (2001). Pesquisa de marketing: uma orientação aplicada (3a ed.). Porto Alegre: Bookman.

Mathieson, S. (1988). Why triangulate? Educational Researcher, 17(2), 13-17.

Maxwell, J. A. (1996). Qualitative research design: an interactive approach. Thousand Oaks: Sage.

Miles, M. B., \& Huberman, A. M. (1994). Qualitative data analysis: an expanded sourcebook (2 ${ }^{\text {nd }}$ ed.). Thousand Oaks: Sage.

Moorman, C., \& Miner, A. S. (1998). The convergence of planning and execution: improvisation in new product development. Journal of Marketing, 62(3), 1-20.

Nunnally, J. C., \& Bernstein, I. H. (1994). Psychometric theory (3rd ed.). New York: McGraw-Hill.

Parasuraman, A., Zeithaml, V. A., \& Berry, L. L. (1985). A conceptual model of service quality and its implications for future research. Journal of Marketing, 49(4), 41-50.

Parasuraman, A., Zeithaml, V. A., \& Berry, L. L. (1988). SERVQUAL: a multiple-item scale for measuring consumer perceptions of service quality. Journal of Retailing, 64(1), 12-40.

Reichheld, F. F. (1996). The loyalty effect: the hidden force behind growth, profits, and lasting value. Boston: Harvard Business School Press.

Reinartz, W. J., \& Kumar, V. (2003). The impact of customer relationship characteristics on profitable lifetime duration. Journal of Marketing, 67(1), 77-99.

Ruekert, R. W., \& Churchill, G. A., Jr. (1984). Reliability and validity of alternative measures of channel member satisfaction. Journal of Marketing Research, 21(2), 226-233.

Rust, R. T., Zeithaml, V. A., \& Lemon, K. N. (2001). O valor do cliente: o modelo que está reformulando a estratégia corporativa. Porto Alegre: Bookman.

Saxe, R., \& Weitz, B. A. (1982). The SOCO scale: a measure or the customer orientation of salespeople. Journal of Marketing, 19(3), 343-351.

Selltiz, C., Wrightsman, L. S., \& Cook, S. W. (1987). Medidas na pesquisa social. In L. H. Kidder (Org.). Métodos de pesquisa nas relações sociais (Vol. 2, 2a ed.). São Paulo: EPU.

Spector, P. E. (1988). Development of the work locus of control scale. Journal of Occupational Psychology, 61(4), 335-340.

Spector. P. E. (1992). Summated rating scale construction. Newbury: Sage Publications.

West, S. G., Finch, J. F., \& Curran, P. J. (1995). Structural equation models with non-normal variables: problems and remedies. In R. H. Hoyle (Ed.). Structural equation modeling: concepts, issues and applications (pp. 56-75). London: Sage Publications. 


\section{APÊNDICE: CONSTRUTOS E INDICADORES DA ESCALA VVBB-ALTA RENDA}

\begin{tabular}{|c|c|c|}
\hline $\begin{array}{l}\text { CONSTRUTO EMPATIA - Capacidade de transmitir uma imagem favorável e } \\
\text { superior da marca, criando identidade com o cliente de forma que ele se sinta } \\
\text { satisfeito, convicto e comprometido em manter o relacionamento com o banco. }\end{array}$ & \begin{tabular}{|c|} 
Alfa de \\
Cronbach: \\
0,62
\end{tabular} & $\begin{array}{c}\text { Cargas } \\
\text { fatoriais } \\
(\lambda)\end{array}$ \\
\hline \multicolumn{2}{|l|}{ 1. As pessoas farão comentários elogiosos, quando se referirem ao Banco Ideal. } & \\
\hline \multicolumn{2}{|l|}{ 2. O Banco Ideal saberá quais são as preferências de cada cliente. } & 78 \\
\hline \multicolumn{2}{|l|}{ 3. O Banco Ideal terá funcionários que gostam de se relacionar com pessoas. } & \\
\hline \multicolumn{2}{|l|}{$\begin{array}{l}\text { 4. Os funcionários do Banco Ideal estarão disponíveis para ajudar a resolver até mesmo as } \\
\text { solicitações mais simples do cliente. }\end{array}$} & 0,578 \\
\hline \multicolumn{2}{|c|}{$\begin{array}{l}\text { 5. No dia do seu aniversário o cliente receberá uma ligação do gerente da sua conta no Banco Ideal, } \\
\text { cumprimentando-o pela data. }\end{array}$} & 0,568 \\
\hline \multicolumn{2}{|l|}{ 6. O Banco Ideal terá produtos e serviços adequados aos diferentes tipos de clientes. } & 0 \\
\hline \multicolumn{2}{|l|}{ 7. O gerente de conta atuará como conselheiro do cliente do Banco Ideal. } & 0 \\
\hline \multicolumn{2}{|l|}{ 8. O cliente do Banco Ideal receberá tratamento semelhante em qualquer uma das agências. } & \\
\hline \multicolumn{2}{|l|}{$\begin{array}{l}\text { 9. Todas as dependências do Banco Ideal seguirão o mesmo padrão de funcionamento, fazendo } \\
\text { dele um banco com identidade única. }\end{array}$} & 0,5 \\
\hline \multicolumn{2}{|l|}{ 10. O Banco Ideal apoiará ações e investimentos de interesse social. } & \\
\hline \multicolumn{2}{|c|}{$\begin{array}{l}\text { 11. As dependências (agências e salas de auto-atendimento) do Banco Ideal terão aspecto visual que } \\
\text { agrade ao cliente. }\end{array}$} & 0,510 \\
\hline $\begin{array}{l}\text { CONSTRUTO 2: CONFIABILIDADE - Demonstrar, de forma permanente, que o } \\
\text { banco é capaz de prestar serviços e disponibilizar produtos que cumprem o } \\
\text { prometido, utilizando-se de linguagem clara, informações fidedignas e } \\
\text { solucionando problemas com presteza e interesse. }\end{array}$ & $\begin{array}{l}\text { Alfa de } \\
\text { Cronbach: } \\
\quad 0,72\end{array}$ & $\begin{array}{l}\text { Cargas } \\
\text { fatoriais } \\
(\lambda)\end{array}$ \\
\hline \multicolumn{2}{|l|}{$\begin{array}{l}\text { 12. Os extratos fornecidos pelo Banco Ideal conterão informações que o cliente entenda como } \\
\text { importantes para ele. }\end{array}$} & 0,676 \\
\hline \multicolumn{2}{|l|}{$\begin{array}{l}\text { 13. Todos os documentos referentes a transações com o Banco Ideal terão linguagem facilmente } \\
\text { compreensível pelo cliente. }\end{array}$} & 0 \\
\hline \multicolumn{2}{|l|}{ 14. O Banco Ideal cumprirá fielmente o que for acordado com o cliente. } & 0,6 \\
\hline \multicolumn{2}{|l|}{$\begin{array}{l}\text { 15. Os funcionários do Banco Ideal saberão utilizar a linguagem mais adequada para se comunicar } \\
\text { com os diferentes tipos de cliente. }\end{array}$} & 0,645 \\
\hline \multicolumn{2}{|l|}{ 16. O Banco Ideal adotará uma linguagem clara, ao se comunicar com o cliente. } & \\
\hline \multicolumn{2}{|l|}{$\begin{array}{l}\text { 17. O Banco Ideal comunicará previamente ao cliente toda mudança que afete a movimentação da } \\
\text { sua conta corrente. }\end{array}$} & 0,63 \\
\hline \multicolumn{2}{|l|}{ 18. As falhas do Banco Ideal serão corrigidas tão logo sejam detectadas. } & \\
\hline \multicolumn{2}{|c|}{ 19. O cliente se sentirá protegido de danos à sua integridade física nas dependências do Banco Ideal. } & 0,568 \\
\hline \multicolumn{2}{|l|}{ 20. Todas as pessoas que precisarem dos serviços do Banco Ideal serão atendidas com respeito. } & 0,551 \\
\hline $\begin{array}{l}\text { CONSTRUTO 3: PREÇO - I } \\
\text { motivando o incremento na ac } \\
\text { banco. Alfa de Cronbach: } 0,56 \text {. }\end{array}$ & \begin{tabular}{|c|} 
Alfa de \\
Cronbach: \\
$\mathbf{0 , 5 6}$
\end{tabular} & $\begin{array}{c}\text { Cargas } \\
\text { fatoriais } \\
(\lambda)\end{array}$ \\
\hline éstimos no Banco Ideal serão inferiores às dos concorren & & 0,701 \\
\hline $\begin{array}{l}\text { 22. Os rendimentos das aplicações financeiras no Banco Ideal serão maiores do que nos } \\
\text { concorrentes. }\end{array}$ & & 0,695 \\
\hline á descontos e prêmios ao cliente pelo maior uso do cartã & & 0 \\
\hline $\begin{array}{l}\text { 24. O Banco Ideal concederá ao cliente um período por mês para utilização do limite } \\
\text { especial sem cobrança de juros. }\end{array}$ & & 0 \\
\hline 25. $\begin{array}{l}\AA n \\
\text { de }\end{array}$ & & 0,53 \\
\hline
\end{tabular}




\begin{tabular}{|c|c|c|}
\hline $\begin{array}{l}\text { CONSTRUTO 4: BENEVOLÊNCIA - Adotar práticas que respeitem e favoreçam } \\
\text { as preferências do cliente, acima dos interesses do banco. }\end{array}$ & $\begin{array}{c}\text { Alfa de } \\
\text { Cronbach: } \\
\mathbf{0 , 6 3}\end{array}$ & $\begin{array}{l}\text { Cargas } \\
\text { fatoriais } \\
(\lambda)\end{array}$ \\
\hline \multicolumn{2}{|c|}{ 26. O Banco Ideal oferecerá seus produtos e serviços, independentemente do interesse do cliente ${ }^{(1)}$. } & $\underline{0,763}$ \\
\hline \multicolumn{2}{|l|}{ 27. $\frac{\text { O Banco Ideal poderá enviar todo tipo de propaganda ao cliente, independentemente da sua }}{\text { vontade }^{(1)}}$} & $\underline{0,725}$ \\
\hline \multicolumn{2}{|l|}{ 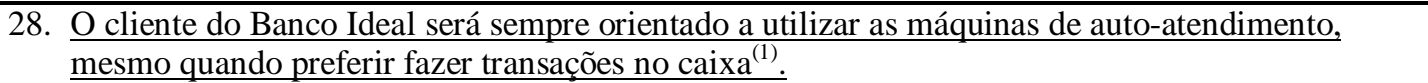 } & $\underline{0,567}$ \\
\hline $\begin{array}{l}\text { CONSTRUTO 5: COMPETENCIA - Demonstrar que o banco possui habilidades, } \\
\text { recursos e compromisso para que os meios que viabilizam as transações com o } \\
\text { cliente funcionem e atuem de forma correta e segura. }\end{array}$ & $\begin{array}{l}\text { Alfa de } \\
\text { Cronbach: } \\
\mathbf{0 , 6 4}\end{array}$ & $\begin{array}{l}\text { Cargas } \\
\text { fatoriais } \\
(\lambda)\end{array}$ \\
\hline \multicolumn{2}{|l|}{ 29. As máquinas de auto-atendimento do Banco Ideal não apresentarão problemas. } & 0,618 \\
\hline \multicolumn{2}{|c|}{$\begin{array}{l}\text { 30. O Banco fará contato com o cliente, quando não houver saldo suficiente na sua conta para pagar } \\
\text { um cheque ou outro tipo de débito. }\end{array}$} & 0,599 \\
\hline \multicolumn{2}{|l|}{ 31. As transações pela Internet com o Banco Ideal estarão protegidas contra fraudes. } & 0,595 \\
\hline \multicolumn{2}{|l|}{ 32. Os extratos emitidos pelo Banco Ideal não conterão erros. } & 0,593 \\
\hline
\end{tabular}

Observações: (1) As variáveis em destaque têm sentido inverso.

Fonte: elaborado pelo autor com base nos dados da pesquisa. 